\title{
A Novel Look-Up Table Design Method for Data Hiding With Reduced Distortion
}

\author{
Xiao-Ping Zhang, Senior Member, IEEE, Kan Li, and Xiaofeng Wang
}

\begin{abstract}
Look-up table (LUT)-based data hiding is a simple and efficient technique to hide secondary information (watermark) into multimedia work for various applications such as copyright protection, transaction tracking or content annotation. This paper studies the distortion introduced by a general LUT-based data hiding. We find that designing LUT according to the distribution of host data and watermark data can greatly reduce the distortion of LUT embedding. A new practical reduced-distortion LUT design method is developed for robust data hiding. The new method is applied in a wavelet domain image data hiding system and only significant wavelet coefficients are used to embed the watermark. A Gaussian mixture model and a related expectation-maximization algorithm-based method are employed to model the statistical distribution of the host image. The statistical model is used to select significant coefficients of the host image for data hiding. The experimental results show that compared to the conventional odd-even LUT embedding method, the presented new LUT data hiding algorithm provides average 1. 5-2. 5 dB PSNR improvement and better robustness for image watermarking.
\end{abstract}

Index Terms-Data hiding, Gaussian mixture model (GMM), JPEG, JPEG2000, look-up table (LUT), robust image watermarking, wavelet transforms.

\section{INTRODUCTION}

D ATA hiding technologies have been widely studied in multimedia security applications, such as copyright protection, transaction tracking, access control, etc. Various watermarking schemes have been proposed to fit the design requirements of various applications [1]. For example, ownership protection requires the watermark, which indicates the ownership, to survive common processing and intentional attacks, and therefore robust watermarking is necessary for such applications. On the other hand, the embedded secondary data can also be used for tampering detection applications, i.e., to determine whether the host media is tampered. Fragile or semi-fragile (robust to common processing, but fragile to intentional attacks) watermarking is suitable for this kind of applications.

Based on embedding mechanisms, existing watermarking schemes can be classified into two categories: host data independent embedding and host data adaptive embedding. In host data independent embedding algorithms, the embedded data

Manuscript received June 30, 2005; revised February 28, 2007. This paper was recommended by Associate Editor I. Ahmad.

X.-P. Zhang and X. Wang are with Department of Electrical and Computer Engineering, Ryerson University, Toronto, ON M5B 2K3, Canada. (e-mail: xzhang@ee.ryerson.ca).

K. Li is with Cantata Technology, Needham, MA 02494 USA.

Color versions of one or more of the figures in this paper are available online at http://ieeexplore.ieee.org.

Digital Object Identifier 10.1109/TCSVT.2008.919088 have no relation to the host data. The additive spread spectrum algorithm [2]-[4] is a representative of this category. In host data adaptive embedding, the embedding scheme depends on the host data visual properties such as in [5], or the data hiding is achieved by deterministically enforcing a relationship between the bits to be embedded and the marked values. Quantization-based watermarking schemes [6]-[8] are in this category. Quantization-based watermarking algorithms embed the watermark, which is often a binary sequence, into the host data by quantizing the host signal with watermark associated quantizers. The look-up table (LUT)-based watermarking algorithm can be treated as a type of quantization-based watermarking. A LUT is a set of quantized values. Each quantized value in a LUT carries either side information "1" or " 0 ." A general LUT watermark embedding algorithm maps the original data to the closest quantized value associated with the desired watermark information bit in a LUT. The embedded watermark can then be extracted from the received image by examining the LUT. The odd-even embedding is a simplest case of LUT embedding, in which the table entries for embedding " 1 " and " 0 " are arranged in an interleaving order. The odd-even embedding is also called run of 1 LUT embedding in [9]. Here run means the largest number of the consecutive 0's or 1's in a LUT. Apparently, the larger is the run, the better is the watermark robustness. It is also indicated in [9] that LUT embedding with larger run constraints introduces larger distortion. This conclusion is based on the assumption that the source data follows a uniform distribution. However, if the original coefficients do not follow a uniform distribution, the probabilities that the coefficients fall into each quantization cell will not be exactly the same. It is then possible that run of 2 LUT embedding may achieve less distortion than the LUT embedding with run 1.

Many wavelet-based watermarking schemes have been proposed for applications such as copyright protection or image authentication [3], [10], [11], since the wavelet transform of images can well represent image features. It is well known that wavelet coefficients do not follow a uniform distribution. In this paper, a new LUT-based watermarking system in the wavelet domain is presented to significantly reduced the distortion without loss of the robustness of the watermark, by carefully designing the LUT according to the statistical distribution of wavelet coefficients.

In the presented new wavelet transform-based image watermarking system, only significant wavelet coefficients (with large magnitude) are selected to bear a watermark, because embedding watermark by modifying these coefficients can survive common image processing [12]. We propose a statistical method based on a Gaussian mixture model (GMM) to select the embeddable coefficients in a wavelet subspace. 


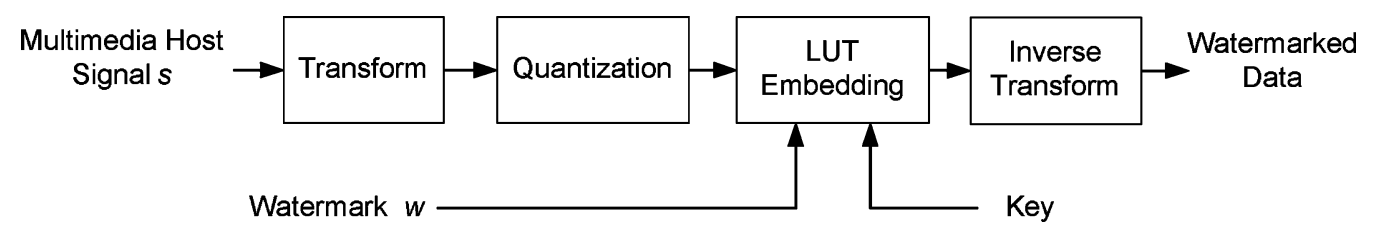

Fig. 1. General LUT embedding algorithm.

The rest of this paper is organized as follows. Section II briefly reviews the LUT-based data hiding. In Section III, the distortion of LUT embedding with run of two is analyzed. In Section IV a practical reduced-distortion LUT design method is proposed. A significant coefficient selection method based on GMM is presented in Section V. Experimental results are presented to demonstrate the advantages of the presented new watermarking scheme over existing schemes in Section VI. Section VII concludes the paper.

\section{OVERVIEW OF THE LUT-BASEd DATA HIDING}

A LUT is a random binary sequence of 0 's and 1's, with limited runs of 0's and 1's. It is the key for the LUT-based watermarking algorithm. Every possible value of the host data is quantized using a quantization function, $Q(\cdot)$, to a small set of quantization values, the number of which is equal to the size of the LUT. The uniform quantizer is the most commonly used quantizer in the LUT-based data hiding schemes. The analysis and discussion of this paper will be based on the uniform quantizer and can be generalized to nonuniform quantizers.

A uniform quantizer with cell width $q$ maps the original signal to $k q, k=1, \cdots, K$, where $K$ is the size of the LUT. Each quantized value, $k q$, in the quantizer carries a watermark bit that is represented by the corresponding $k$ th entry in the LUT. That is to say, to embed a "1" in a host data coefficient, the coefficient is mapped to the nearest quantized value whose corresponding LUT entry is " 1 ." The embedding of a " 0 " is similar.

For simplicity of further analysis, we define the look-up function, Lookup $(\cdot)$, which simply returns a "0" or " 1 " depending on the input index

$$
\operatorname{Lookup}(k q)=\text { value in LUT at index } k \text {. }
$$

We further define the $\operatorname{LUT}(\cdot)$ function, which takes the value of the original signal as the input and maps it to a " 0 " or " 1 " depending on the LUT. The $\operatorname{LUT}(\cdot)$ function is actually a simple composition of the lookup and the quantization function:

$$
\operatorname{LUT}(x)=\operatorname{Lookup}(Q(x)) .
$$

Fig. 1 shows the general process of LUT watermark embedding algorithm. An orthogonal transformation $T_{w}$ decomposes the host data $s$ into coefficients $x$ in the watermark domain to which the watermark $w$ is embedded. Then the coefficients are quantized and mapped according to LUT.

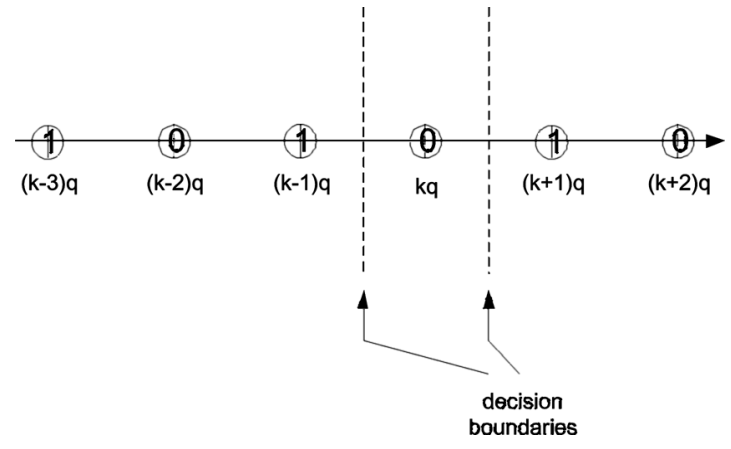

Fig. 2. Odd-even embedding.

The watermark embedding process in the transform domain can be abstracted into the following formula where $s$ is the original coefficient, $x$ is the marked one, $b$ is the bit to be embedded

$$
x= \begin{cases}Q(s), & \text { if } \operatorname{LUT}(s)=b \\ Q(s+d), & \text { if } \operatorname{LUT}(s) \neq b, \text { where } \\ & d=\arg \min _{d}\{|d|, \operatorname{LUT}(s+d)=b\} .\end{cases}
$$

For LUT embedding, once the LUT is known to the detector, the watermark can be extracted easily through a simple lookup from the LUT, i.e.,

$$
\hat{b}=\operatorname{LUT}(\hat{x})
$$

where $\hat{b}$ is the extracted bit and $\hat{x}$ is the possibly corrupted watermarked signal.

A typical LUT embedding algorithm is the odd-even embedding. First, a uniform quantization function $Q(\cdot)$ is defined, which partitions the signal space $\Re$ into quantization subsets as illustrated in Fig. 2. The host data is mapped to the nearest even number quantization point to embed a "0" and the nearest odd number quantization point to embed a "1." Thus, a relationship between the watermark bit and the marked signal is deterministically formed.

The watermark bit is extracted by the following simple way

$$
\hat{b}= \begin{cases}0, & \text { if } \frac{Q(\hat{x})}{q} \text { is even } \\ 1, & \text { if } \frac{Q(\hat{x})}{q} \text { is odd. }\end{cases}
$$

In the odd-even embedding scheme, the table entries for embedding " 0 " and " 1 " are arranged in an interleaving order, $\{\ldots, \operatorname{Lookup}((k-1) q)=0, \operatorname{Lookup}(k q)=$ $1, \operatorname{Lookup}((k+1) q)=0, \operatorname{Lookup}((k+2) q)=1, \ldots\}$, which is also formulated as run $=1$ LUT embedding in [9]. It is also pointed out in [9] that the LUT embedding with larger run constraints introduces larger distortion but has better robustness and thus smaller probability of detection error. 
TABLE I

All Possible CASES FOR ENTRIES OF $(k-1) q, k q$ AND $(k+1) q$ IN A LUT WITH RUN OF 2

\begin{tabular}{|c|c|c|c|}
\hline$(k-1) q$ & $k q$ & $(k+1) q$ & \\
\hline 0 & 0 & 1 & Case 1 \\
\hline 0 & 1 & 0 & Case 3 \\
\hline 0 & 1 & 1 & Case 2 \\
\hline 1 & 0 & 0 & Case 2 \\
\hline 1 & 0 & 1 & Case 3 \\
\hline 1 & 1 & 0 & Case 1 \\
\hline
\end{tabular}

\section{Distortion ANALYSIS FOR LUT BASED DATA Hiding}

During the LUT embedding, when Lookup $(Q(x))$ does not match the watermark bit $b$ to be embedded, a nearby entry in the LUT that is mapped to $b$ is used to represent (quantize) $x$. As such, the run of " 1 " and " 0 " entries of a LUT is related to excessive distortion of the host data other than the nominal quantization error. In this paper, we will specifically analyze the distortion for the LUT with the maximum run of 2 .

In the LUT embedding, the uniform quantization $Q(\cdot)$ divides the signal space into $K$ levels of equal spacing. Then if the $k$ th watermarking entry of LUT is $b$, the data samples of host signal $s$ in the quantization cell of $[(k-1 / 2) q,(k+1 / 2) q]$, which is used to carry watermark bit $b$, are quantized to $k q$. The mean square distortion produced by the uniform quantizer is calculated as

$$
D_{k q}(s)=\int_{(k-1 / 2) q}^{(k+1 / 2) q}|s-k q|^{2} f(s) d s
$$

where $f(s)$ is the probability density function (pdf) of $s$. However, if the desired watermark bit for $s$ is not $b$, the host data must be mapped to the nearest quantization point, $(k \pm l) q, l>0$, corresponding to the desired watermark bit $b$. If the maximum allowable run is 2 , the entries carrying watermark bit $b$ next to the $k$ th entry may be the $(k-1)$ th entry or $(k+1)$ th entry or both. The above situation includes three cases.

Case 1) Left run is 2, i.e., $\operatorname{Lookup}((k-1) q)=$ $\operatorname{Lookup}(k q)=b$, and $\operatorname{Lookup}(k q) \neq$ $\operatorname{Lookup}((k+1) q)$;

Case 2) Right run is 2, i.e., $\operatorname{Lookup}((k-1) q) \neq$ $\operatorname{Lookup}(k q)$, and $\operatorname{Lookup}(k q)=\operatorname{Lookup}((k+$ 1) $q)=b$

Case 3) Run is 1, i.e., $\operatorname{Lookup}((k-1) q) \neq \operatorname{Lookup}(k q)=b$ and $\operatorname{Lookup}(k q)=b \neq \operatorname{Lookup}((k+1) q)$.

All possible cases are illustrated in Table I.

The distortion in each case is analyzed as follows. For Case 1, the samples of $s$ in the quantization cell of $[(k-1 / 2) q,(k+$ $1 / 2) q]$ need to be quantized to $(k+1) q$. The distortion is

$$
\begin{aligned}
D_{\text {Case1 }}(s)= & \int_{(k-1 / 2) q}^{(k+1 / 2) q}|s-(k+1) q|^{2} f(s) d s \\
= & D_{k q}+q^{2} \int_{(k-1 / 2) q}^{(k+1 / 2) q} f(s) d s \\
& -2 q \int_{(k-1 / 2) q}^{(k+1 / 2) q}(s-k q) f(s) d s
\end{aligned}
$$

where $D_{k q}$ is the uniform quantization distortion shown in (6).
Similarly, for Case 2, the samples in $k$ th cell need to be mapped to $(k-1) q$

$$
\begin{aligned}
& D_{\text {Case2 }}(s) \\
& =D_{k q}+q^{2} \int_{(k-1 / 2) q}^{(k+1 / 2) q} f(s) d s+2 q \int_{(k-1 / 2) q}^{(k+1 / 2) q}(s-k q) f(s) d s .
\end{aligned}
$$

For Case 3, there are two nearest quantization points $(k+1) q$ and $(k-1) q$ corresponding to the desired bit simultaneously. Then the original data points in the range of $[(k-1 / 2) q, k q]$ will be quantized to $(k-1) q$, and the data points in the other half interval $[k q,(k+1 / 2) q]$ are quantized to $(k+1) q$. The distortion is composed of two parts

$$
\begin{aligned}
D_{\text {Case3 }}(s)= & \int_{(k-1 / 2) q}^{k q}[s-(k-1) q]^{2} f(s) d s \\
& +\int_{k q}^{(k+1 / 2) q}[s-(k+1) q]^{2} f(s) d s \\
= & D_{k q}+q^{2} \int_{(k-1 / 2) q}^{(k+1 / 2) q} f(s) d s \\
& +2 q\left[\int_{(k-1 / 2) q}^{k q}(s-k q) f(s) d s\right] \\
& \left.\int_{k q}^{(k+1 / 2) q}(s-k q) f(s) d s\right] .
\end{aligned}
$$

If the host data are approximately uniformly or symmetrically distributed within each cell, the last terms of (7) and (8) are close to 0 , and the last term of (9) will be in the range of $\left[-q^{2} \int_{(k-1 / 2) q}^{(k+1 / 2) q} f(s) d s, 0\right]$, and approximately $\left(-q^{2} / 2\right) \int_{(k-1 / 2) q}^{(k+1 / 2) q} f(s) d s$.

For a binary data hiding system, the host data can be divided into two categories: the data points used to embed bit " 0 ," denoted by $s_{0}$, and the data points used to embed bit " 1 ," denoted by $s_{1}$. Assume the pdfs of $s_{0}$ and $s_{1}$ are $f_{0}\left(s_{0}\right)$ and $f_{1}\left(s_{1}\right)$, respectively. First, we consider the overall mean squared distortion due to quantization only

$$
\begin{gathered}
\mathrm{MSE}_{q}=\sum_{k=1}^{K}\left[\int_{(k-1 / 2) q}^{(k+1 / 2) q}\left|s_{0}-k q\right|^{2} f_{0}\left(s_{0}\right) d s_{0}\right. \\
\left.+\int_{(k-1 / 2) q}^{(k+1 / 2) q}\left|s_{1}-k q\right|^{2} f_{1}\left(s_{1}\right) d s_{1}\right] \\
=\sum_{k=1}^{K}\left[D_{k q}\left(s_{0}\right)+D_{k q}\left(s_{1}\right)\right] .
\end{gathered}
$$

Now consider $K$ binary entries in the LUT. In all $K$ quantization cells, some data are mapped to their own local reconstruction points. Other data have to be mapped to neighboring 
reconstruction points where the above three cases happen. According to (7)-(9), the overall LUT embedding distortion can be formulated as

$$
\begin{aligned}
\mathrm{MSE}_{w}= & \mathrm{MSE}_{q}+q^{2} \sum_{k=1}^{K} \alpha_{0, k} \int_{(k-1 / 2) q}^{(k+1 / 2) q} f_{0}\left(s_{0}\right) d s_{0} \\
& +q^{2} \sum_{k=1}^{K} \alpha_{1, k} \int_{(k-1 / 2) q}^{(k+1 / 2) q} f_{1}\left(s_{1}\right) d s_{1} \\
& -\frac{q^{2}}{2} \sum_{k=1}^{K} \beta_{0, k} \int_{(k-1 / 2) q}^{(k+1 / 2) q} f_{0}\left(s_{0}\right) d s_{0} \\
& -\frac{q^{2}}{2} \sum_{k=1}^{K} \beta_{1, k} \int_{(k-1 / 2) q}^{(k+1 / 2) q} f_{1}\left(s_{1}\right) d s_{1}
\end{aligned}
$$

where $\alpha$ and $\beta$ can be 0 or $1, \alpha_{0, k}=1$ if the $k$ th LUT entry is 1 , similarly $\alpha_{1, k}=1$ when the $k$ th LUT entry is 0 , hence $\alpha_{0, k}=$ $1-\alpha_{1, k}$. Only in Case 3, $\beta$ is equal to 1, i.e., if $\operatorname{Lookup}((k-$ 1) $q)=\operatorname{Lookup}((k+1) q) \neq \operatorname{Lookup}(k q)=1, \beta_{0, k}=1$; and if $\operatorname{Lookup}((k-1) q)=\operatorname{Lookup}((k+1) q) \neq \operatorname{Lookup}(k q)=0$, $\beta_{1, k}=1$. Note that $\beta_{0, k}=\alpha_{1, k-1} \alpha_{0, k} \alpha_{1, k+1}$ and $\beta_{1, k}=$ $\alpha_{0, k-1} \alpha_{1, k} \alpha_{0, k+1}$.

Let $P_{0, k}$ and $P_{1, k}$ represent the probabilities that $s_{0}$ and $s_{1}$ fall into the $k$ th quantization cell of $Q(\cdot)$, respectively, i.e.,

$$
P_{0, k}=\int_{(k-1 / 2) q}^{(k+1 / 2) q} f_{0}\left(s_{0}\right) d s_{0}, P_{1, k}=\int_{(k-1 / 2) q}^{(k+1 / 2) q} f_{1}\left(s_{1}\right) d s_{1} .
$$

We can rewrite (11) as

$$
\begin{aligned}
\operatorname{MSE}_{w}=\mathrm{MSE}_{q} & +q^{2} \sum_{k=1}^{K} \alpha_{0, k} P_{0, k}+q^{2} \sum_{k=1}^{K} \alpha_{1, k} P_{1, k} \\
& -\frac{q^{2}}{2} \sum_{k=1}^{K} \beta_{0, k} P_{0, k}-\frac{q^{2}}{2} \sum_{k=1}^{K} \beta_{1, k} P_{1, k} .
\end{aligned}
$$

If the original host signal follows a uniform distribution, the probabilities that the signal falls into each quantization cell will be exactly the same, then different LUTs with the same run have the same overall distortion. However, most real-world signals are not uniformly distributed. For example, the wavelet coefficients of a natural image do not follow a uniform distortion. The probabilities that a nonuniform signal point falls in each quantization cell are different from each other. Embedding watermarks according to different LUT schemes can produce different distortions. From (13), it can be seen that the parameters $\alpha_{0, k}, \alpha_{1, k}, \beta_{0, k}$ and $\beta_{1, k}, k=1, \cdots, K$, corresponding to each LUT scheme are unique. Therefore, various distortions can be obtained with various LUT schemes. The objective is to find the LUT that achieves the minimum distortion.

\section{Reduced-Distortion LUT Design Algorithm}

Fig. 3 illustrates an example of wavelet coefficients that are used to embed the watermark. The numbers of coefficients used to embed the watermark " 0 " and " 1 " falling into each quantization cell are different from each other, and represented by the

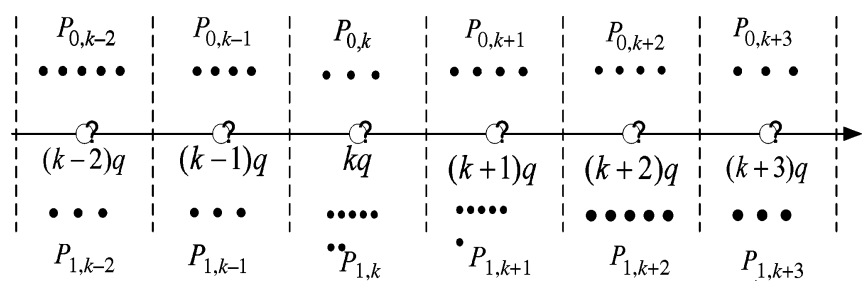

Fig. 3. Minimum-distortion LUT needs to be generated according to the given wavelet coefficient distribution.

numbers of dots shown in Fig. 3. Among a variety of LUTs with run of 2 , our design objective is to find the one that achieves minimum distortion.

According to (13), the LUT embedding distortion is determined by the LUT ( $\alpha$ and $\beta$ ) and the distribution of $s_{0}$ and $s_{1}$. The reduced-distortion LUT is obtained by carefully examining the probabilities that the coefficients used to embed " 0 " and " 1 " fall into each cell, $P_{0, k}$ and $P_{1, k}, k=1,2 \ldots K$. In practice, the probabilities can be approximated by calculating the frequency of the appearance of coefficients falling in cell $k$. For the $k$ th cell in a LUT with run of 2 , either the coefficients used to embed " 0 " or the coefficients used to embed " 1 " are mapped to $k q$, and the rest coefficients are mapped to the neighboring quantization points $(k-1) q$ or $(k+1) q$. Different LUT strategies determine the mapping from coefficients to quantization points and generate different embedding distortions. The difference between the pair of probabilities, $\mathrm{PD}_{k}=P_{0, k}-P_{1, k}$, determines the distortion difference between the case of the $k$ th LUT entry being assigned bit " 0 " and that of " 1 ," shown as follows.

From (13), note that the embedding distortion generated by the $k$ th LUT entry, denoted by $\operatorname{MSE}_{w}(k)$, is

$$
\begin{aligned}
\operatorname{MSE}_{w}(k)=\alpha_{0, k} P_{0, k} q^{2}+ & \alpha_{1, k} P_{1, k} q^{2} \\
& -\beta_{0, k} P_{0, k} \frac{q^{2}}{2}-\beta_{1, k} P_{1, k} \frac{q^{2}}{2} .
\end{aligned}
$$

The difference between the distortions generated by setting $k$ th LUT entry to " 0 " (when $\alpha_{1, k}=1$ ) and " 1 " (when $\alpha_{0, k}=1$ ) is, therefore

$$
\begin{aligned}
\operatorname{MSE}_{w, d}(k)= & \left(P_{1, k} q^{2}-\beta_{1, k} P_{1, k} \frac{q^{2}}{2}\right) \\
& -\left(P_{0, k} q^{2}-\beta_{0, k} P_{0, k} \frac{q^{2}}{2}\right) \\
= & \left(P_{1, k}-P_{0, k}\right) q^{2} \\
& -\left(\beta_{1, k} P_{1, k}-\beta_{0, k} P_{0, k}\right) \frac{q^{2}}{2} .
\end{aligned}
$$

In general (recall Table I), the first term of (15) is dominant. That is, the larger is $\left|\mathrm{PD}_{k}\right|$, the less distortion it may be if the entry is set to the bit with the less probability. Then each entry should be set to the bit corresponding to the higher probability of the pair of probabilities with larger probability difference. According to this principle, the reduced-distortion LUT design can be obtained as follows.

First the values of $\left|\mathrm{PD}_{k}\right|$ are calculated, sorted in a descending order and stored in a queue. Then the LUT is built 


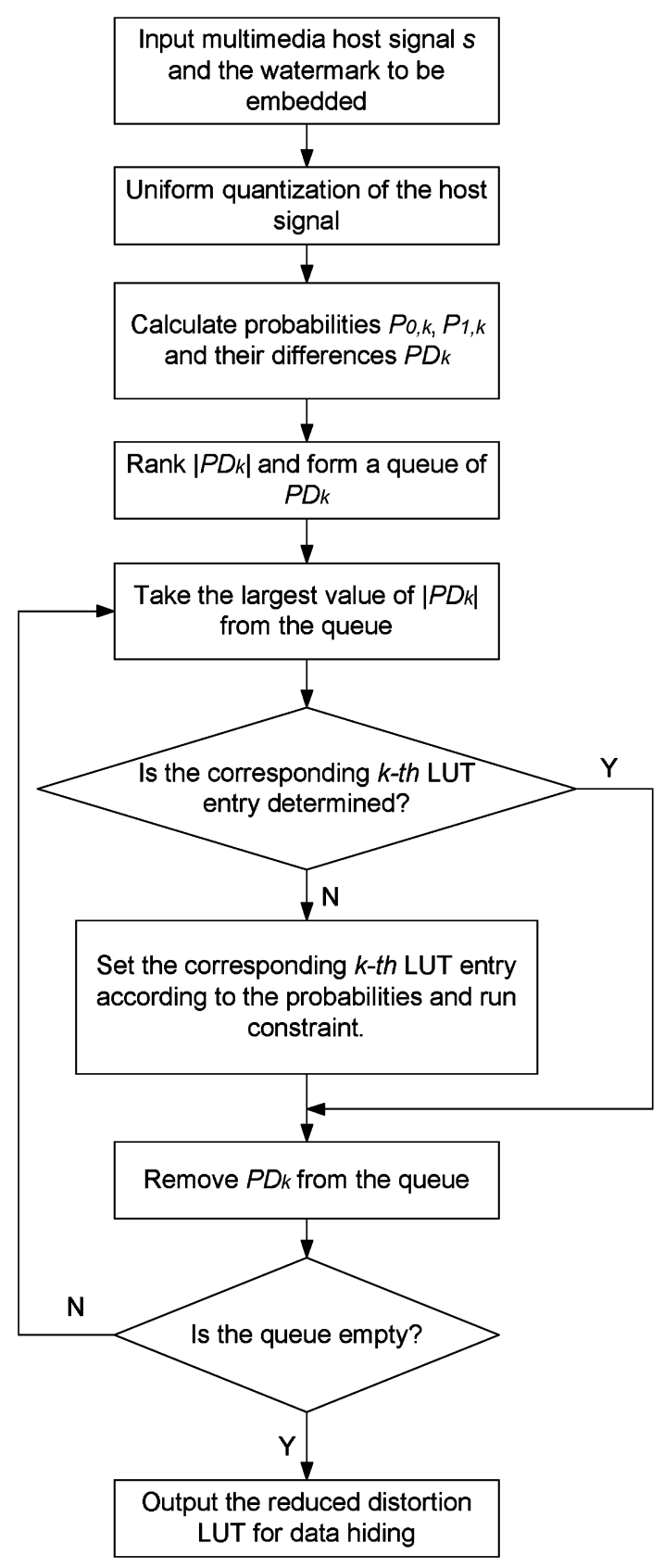

Fig. 4. Flowchart of the reduced-distortion LUT algorithm.

in the way that the entry corresponding to the largest probability difference are set to the bit corresponds to the larger probability of that pair of probabilities. For example, if $\left|\mathrm{PD}_{k}\right|$ is the largest in the queue and the LUT entry Lookup $(k q)$ is not determined, we set $\operatorname{Lookup}(k q)=0$ if $P_{0, k}>P_{1, k}$, otherwise $\operatorname{Lookup}(k q)=1$. After each operation, the largest probability difference is removed from the queue and the next largest probability difference is processed in the same fashion. The rule is that the maximum run constraint should be satisfied all the time, i.e., for maximum run constraint of 2 , the run for " 0 " or " 1 " must be equal or less than 2 . The algorithm for run of 2 can be summarized in the following steps.

Step 1) Calculate the difference, $\mathrm{PD}_{k}$, between each pair of probabilities, i.e., $\mathrm{PD}_{k}=P_{0, k}-P_{1, k}$.

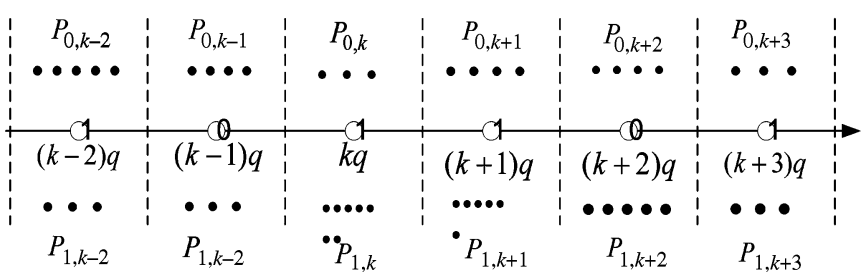

Fig. 5. Example of a reduced-distortion LUT with the maximum run constraint of 2 .

Step 2) Rank the absolute value of $\mathrm{PD}_{k}$ in a descending order.

Step 3) Find the largest absolute value of $\mathrm{PD}_{k}$ from the above queue. If $\operatorname{Lookup}(k q)$ has been determined, go to STEP 4. Otherwise, among the determined LUT entries, if the maximum run of 2 has already been reached, i.e., $\operatorname{Lookup}((k-2) q)=\operatorname{Lookup}((k-1) q)=l$ or $\operatorname{Lookup}((k-1) q)=\operatorname{Lookup}((k+1) q)=l$ or $\operatorname{Lookup}((k+1) q)=\operatorname{Lookup}((k+2) q)=l$, $l \in\{0,1\}, \operatorname{Lookup}(k q)$ is set to the complement of $l: \operatorname{Lookup}(k q)=\bmod (l-1,2)$, regardless of $j$ value; otherwise $\operatorname{Lookup}(k q)=b$, where $b=\arg \max _{j} P_{j, k}$.

Step 4) $\mathrm{PD}_{k}$ is removed from the queue. If the queue is not empty, go back to STEP 3. Otherwise, all LUT entries have been assigned and the reduced-distortion LUT is obtained.

Fig. 4 illustrates the flowchart of the above reduced-distortion LUT generation algorithm. An illustration is shown in Fig. 5.

Note that the LUT is signal dependent and needs to be sent to the detector for watermark extraction. Such additional side information transmission may limit application of the presented method. However, the size of such side information is usually small. In some image compression standards such as JPEG, such LUT quantization tables may be stored in header information. In addition, with a little flexibility, the presented scheme can be used to generate more than one reduced-distortion LUTs. The signal dependent LUT in itself can then be used as a secret key to enhance the security of the embedding and may be desirable in many applications.

\section{Significant Coefficient Selection Based on A Gaussian Mixture Model IN THE WaVELET DOMAIN}

In the new scheme, only wavelet coefficients with large magnitude are selected to bear the watermark since, in general, these coefficients have little change after image processing and compression attacks. A statistical method is designed to select the embeddable coefficients based on a GMM in the wavelet domain. The wavelet coefficients have a peaky, heavy-tailed marginal distribution [13], [14]. Only a few significant coefficients take large values at the positions of singularities such as edges, while most others take small values. This statistical characteristic can be expressed by using a two component Gaussian mixture

$$
\begin{aligned}
p\left(w_{i}\right) & =p_{s} \cdot g\left(w_{i}, 0, \sigma_{s}^{2}\right)+p_{l} \cdot g\left(w_{i}, 0, \sigma_{l}^{2}\right) \\
p_{s}+p_{l} & =1 .
\end{aligned}
$$




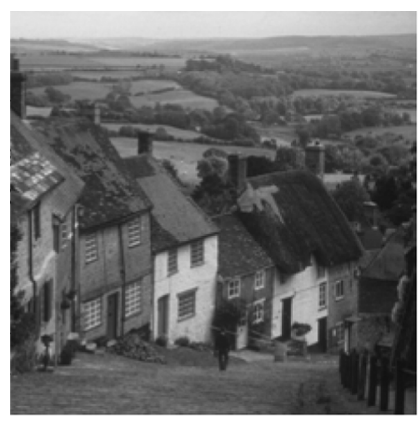

(a)

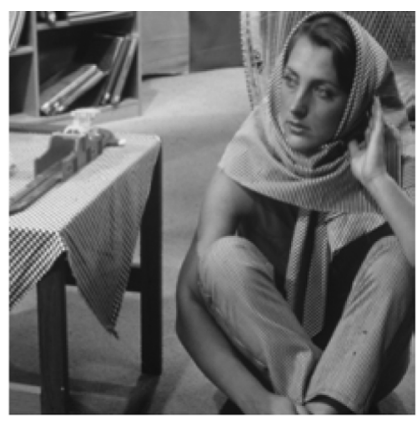

(b)

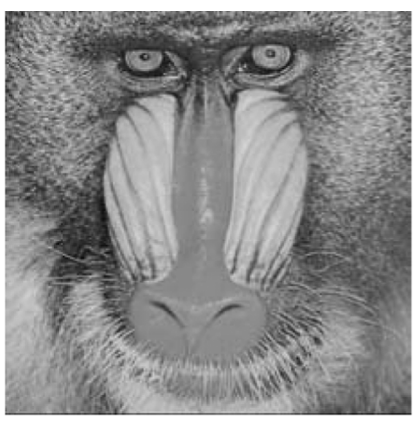

(c)

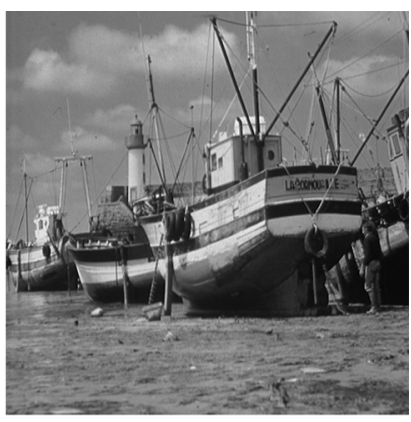

(d)

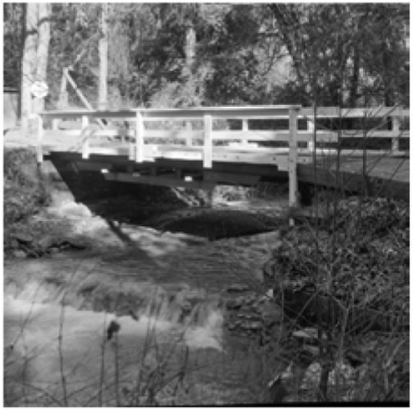

(e)

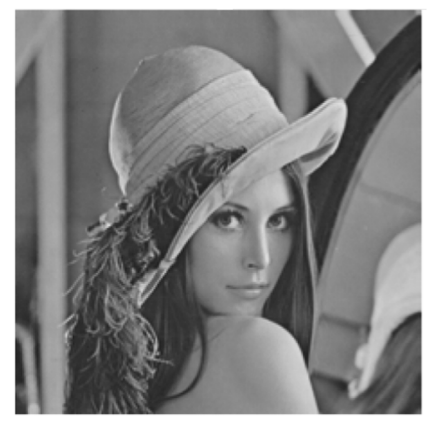

(f)

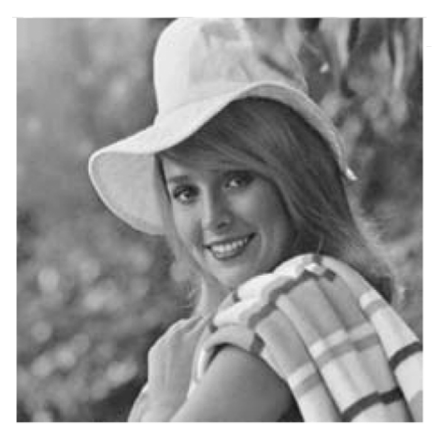

(g)

Fig. 6. Test images. (a) Golden Hill. (b) Barbara. (c) Baboon. (d) Boat. (e) Bridge. (f) Lena. (g) Elaine.

where the class of small coefficients is represented by subscript " $s$ " and the class of large coefficients by subscript " $l . "$ The a priori probabilities of the two classes are represented by $p_{s}$ and $p_{l}$, respectively. The Gaussian component for the small coefficients has a relatively small variance $\sigma_{s}^{2}$, capturing the peakiness around zero, while the component for the large state has a relatively large variance $\sigma_{s}^{2}$, capturing the heavy tails. An EM algorithm can be employed to identify the GMM by obtaining the model parameters $\left[p_{s}, p_{l}, \sigma_{s}^{2}, \sigma_{l}^{2}\right]$.

The watermark is only embedded into the class of large coefficients because modifying coefficients which represent the edge information will introduce less perceptual degradation. The GMM is used to find large coefficients for watermarking by classifying the coefficients into large coefficient class and small coefficient class. In practice, assume there are $m$ coefficients in the wavelet space, the number of large coefficients is approximately $m \cdot p_{l}$ and the number of small coefficients is approximately $m \cdot p_{s}$. The largest $m \cdot p_{l}$ coefficients are therefore selected for watermark embedding. At the detector end, the large coefficient map can be transmitted to the detector or regenerated by reconstructing the GMM. Note that the reconstruction of the GMM may suffer some errors.

While such signal dependent selective embedding improves the watermarking embedding performance in terms of robustness and perceptional quality, the desynchronization is a common problem for most signal dependent watermarking schemes. The synchronization problem may be solved by incorporating erasures and errors correcting codes as shown in [15]. The detailed discussion of the erasures and errors correcting codes is beyond the scope of our paper.

\section{Simulations}

The presented new data hiding/watermarking scheme is tested on seven commonly used images of different types,

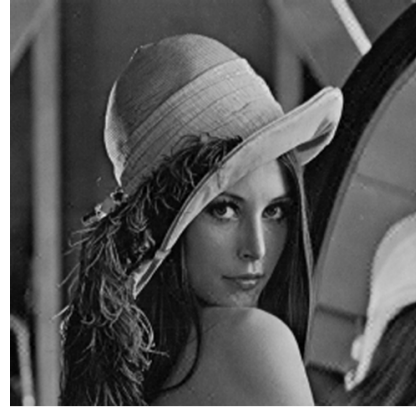

(a)

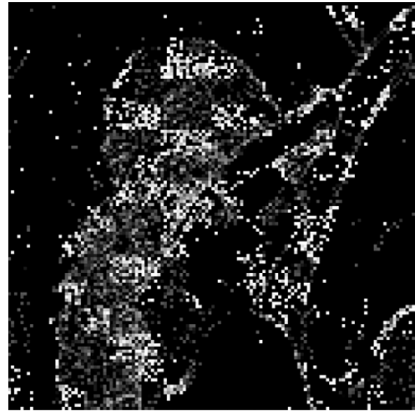

(b)
Fig. 7. Watermarked image and the difference from the original image with black denoting zero difference.

namely, Golden Hill, Barbara, Baboon, Boat, Bridge, Lena, and Elaine, as shown in Fig. 6. First binary watermark is embedded into the images by applying the new method. Fig. 7 shows one example (Lena). The modified significant coefficients are mainly at the edges (singularities) of the image. The watermark robustness to common operations such as image compression is tested. The discrete cosine transform (DCT)-based coding system, JPEG baseline, and the discrete wavelet transform (DWT)-based coding system, JPEG 2000, are the two compression attacks used in our tests.

The quality of watermarked and attacked images is evaluated by peak-signal-to-noise-ratio (PSNR). The watermark robustness under several intentional/unintentional attacks is evaluated by bit-error rate (BER). The watermark robustness by the average BER is evaluated for all test images. As shown in Fig. 8, the BER of the extracted watermark is larger than $75 \%$ until the compression quality factor is smaller than 60 . 


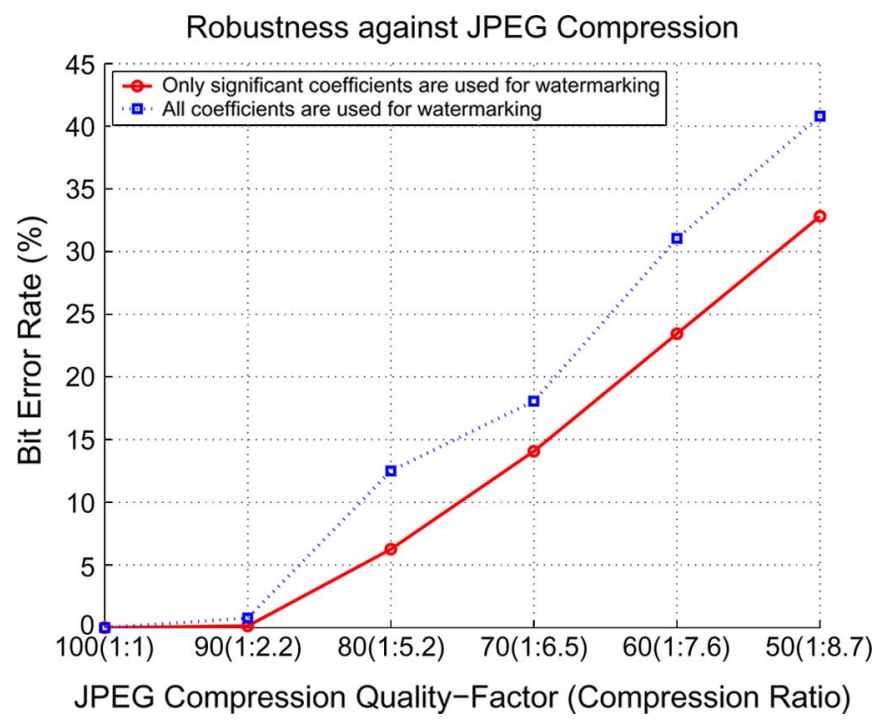

Fig. 8. Watermark robustness for JPEG compression.

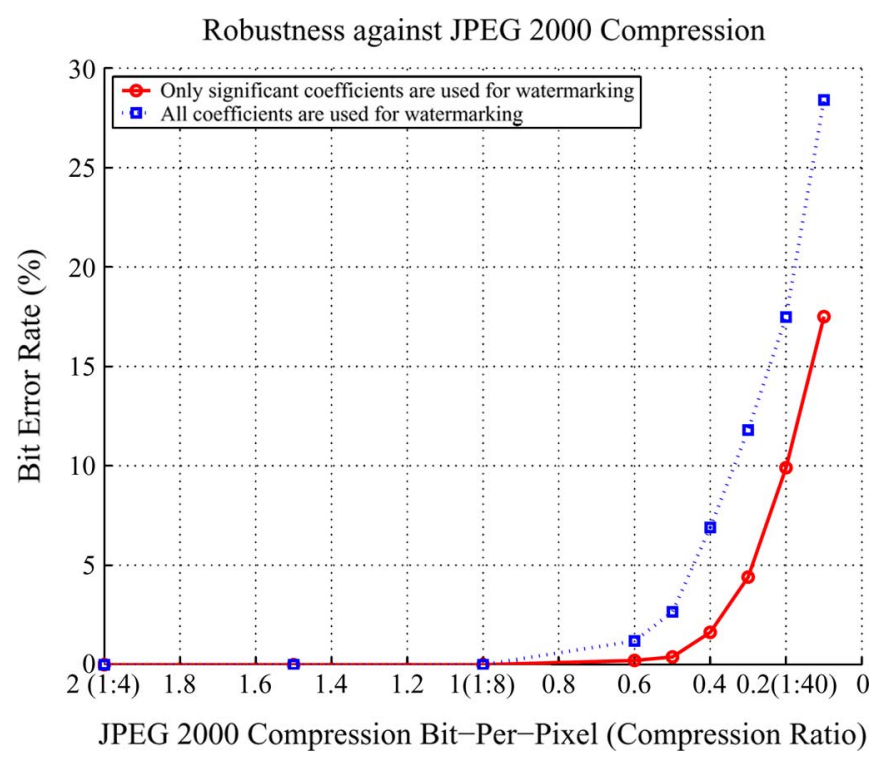

Fig. 9. Watermark robustness for JPEG 2000 compression.

Since the watermark is embedded in the wavelet domain, the presented algorithm has perfect robustness against the DWTbased JPEG 2000 compression attack when the compression is relatively less. The results are shown in Fig. 9. The decoded watermark can be $100 \%$ reconstructed after JPEG 2000 compression of $1 \mathrm{bpp}$ and is reliable until the compression bit-rate smaller than $0.2 \mathrm{bpp}$ (1:40). The embedded watermark and the extracted watermark in Fig. 10 is an example showing that the new scheme can survive severe JPEG 2000 compression (1:40) very well. The comparison of watermark embedding with and without the statistical model based significant coefficient selection is also shown in the Figs. 8 and 9. The advantages of the new method for the coefficient selection are apparent.

Finally, the average host data distortion of all LUTs with maximum allowable run of 2 , the host data distortions of the interleaving LUT (odd-even embedding, run $=1$ ) and that of the reduced-distortion LUT are compared in terms of various

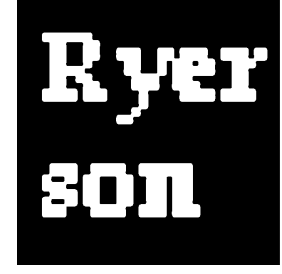

(a)

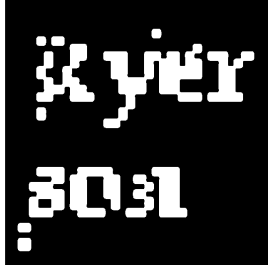

(b)
Fig 10. (a) Embedded watermark and (b) the extracted watermark from JPEG 2000 severely compressed (with compression ratio 1:40) image.

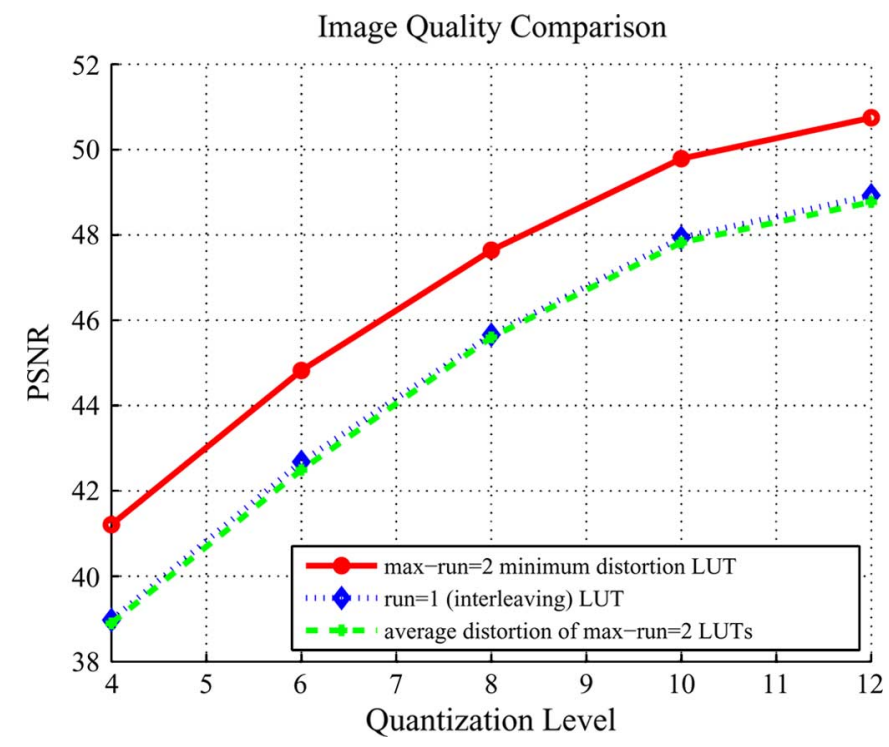

Fig. 11. The image quality (distortion) comparison: the average host data distortion of all LUTs with maximum allowable run of 2, the host data distortions of the interleaving LUT (odd-even embedding, run $=1$ ) and that of the reduced-distortion LUT.

quantization levels. According to Fig. 11, the distortion of the new method is the minimum. Note that additional experiments in more images showed similar performance results.

\section{CONCLUSION}

We have analytically evaluated the distortion brought by LUT-based data hiding with the run constraints. Based on this analysis, it is shown that besides the nominal quantization distortion, data hiding schemes generate additional distortions on host data. This additional distortion is related to the host data and watermark data distributions. Based on the formulated relationship, a novel algorithm is presented to design LUT which can achieve reduced-distortion on the host signal and improve the watermarked signal quality without loss of the watermark robustness. If the security issue is taken into account, with a little flexibility, the presented scheme can be used to generate more than one reduced-distortion LUTs. The LUT can serve as a security key. Experimental results confirm the theoretical distortion analysis and show that the reduced-distortion LUT obtained with our method is superior to the odd-even (interleaving) LUT data embedding in terms of image quality and robust to common image processing such as compression. Nevertheless, we note that the distortion optimization of the 
LUT-based data hiding is a highly nonlinear problem. The presented reduced-distortion LUT has not achieved the true minimum distortion. Our future work will include extending the algorithm to multiple runs (more than two) and finding feasible algorithms to optimize the LUT distortion.

\section{REFERENCES}

[1] B. B. Zhu, M. D. Swanson, and A. H. Tewfik, "When seeing isn't believing," IEEE Signal Process. Mag., vol. 21, no. 2, pp. 40-49, Mar. 2004.

[2] J. Cox, F. T. Leighton, and T. Shamoon, "Secure spread spectrum watermarking for multimedia," IEEE Trans. Image Process., vol. 6, pp. 1673-1687, 1997.

[3] X.-G. Xia, C. G. Boncelet, and G. R. Arce, "A multiresolution watermark for digital images," in Proc. Int. Conf. Image Process., Santa Barbara, CA, Oct. 26-29, 1997, vol. III, pp. 548-551.

[4] F. Hartung, J. K. Su, and B. Girod, "Spread spectrum watermarking: Malicious attacks and counterattacks," in Proc. SPIE Security Watermarking Multimeida Contents, Jan. 1999, vol. 3657.

[5] C. Podilchuk and W. Zeng, "Image-adaptive watermarking using visual models," IEEE J. Sel. Areas Commun., vol. 16, no. 4, pp. 525-539, May 1998.

[6] B. Chen and G. W. Wornell, "Quantization index modulation: A class of provably good methods for digital watermarking and information embedding," IEEE Trans. Inf. Theory, vol. 47, no. 5, pp. 1423-1443, May 2001.

[7] B. Chen and G. W. Wornell, "An information-theoretic approach to the design of robust digital watermarking systems," in Proc. Int. Conf. Acoust., Speech, Signal Process., 1999, vol. 4, pp. 2061-2064.

[8] D. Kundur and D. Hatzinakos, "Digital watermarking for telltale tamper proofing and authentication," Proc. IEEE, vol. 87, no. 7, pp. 1167-1180, Jul. 1999.

[9] M. Wu, "Joint security and robustness enhancement for quantization based data embedding," IEEE Trans. Circuits Syst. Video Technol., vol. 13, no. 8, pp. 831-841, Aug. 2003.

[10] H. J. Wang and C. C. J. Kuo, "A integrated approach to embedded image coding and watermarking," in Proc. Int. Conf. Acoust., Speech, Signal Process., May 12-15, 1998, vol. 6, pp. 3721-3724.

[11] D. Kundur and D. Hatzinakos, "A robust digital image watermarking method using wavelet-based fusion," in Proc. Int. Conf. Image Process., Oct. 26-29, 1997, vol. 1, pp. 544-547.

[12] P. Su, H. M. Wang, and C. C. J. Kuo, "An integrated approach to image watermarking and JPEG-2000 compression,” J. VLSI Signal Process., vol. 27, pp. 35-53, 2001.

[13] J. Romberg, H. Choi, and R. Baraniuk, "Bayesian tree-structured image modeling using wavelet-domain hidden markov models," IEEE Trans. Image Process., vol. 10, no. 7, Jul. 2001.

[14] H. Yuan and X.-P. Zhang, "Fragile watermark based on the gaussian mixture model in wavelet domain for image authentication," in Proc. Int. Conf. Image Process., Sep. 14-17, 2003, vol. 1, pp. 505-508.
[15] K. Solanki, N. Jacobsen, U. Madhow, B. Manjunath, and S. Chandrasekaran, "Robust image-adaptive data hiding using erasure and error correction," IEEE Trans. Image Process., vol. 13, no. 12, pp. 1627-1639, Dec. 2004.

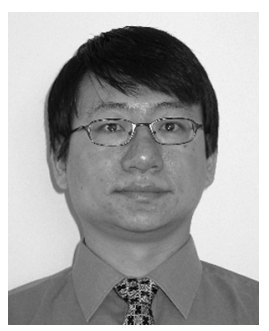

Xiao-Ping Zhang received the B.S. and Ph.D. degrees from Tsinghua University, Beijing, China, in 1992 and 1996, respectively, all in electronic engineering.

Since Fall 2000, he has been with the Department of Electrical and Computer Engineering, Ryerson University, Toronto, ON, Canada, where he is currently Associate Professor and director of Communication and Signal Processing Applications Laboratory (CASPAL). Prior to joining Ryerson, from 1996 to 1998, he was a Postdoctoral Fellow at the University of Texas, San Antonio, and then at the Beckman Institute, the University of Illinois at Urbana-Champaign. He held research and teaching positions at the Communication Research Laboratory, McMaster University, in 1999. From 1999 to 2000, he was a Senior DSP Engineer at SAM Technology, Inc., San Francisco, CA, and a Consultant at San Francisco Brain Research Institute. His research interests include signal processing for communications, multimedia data hiding, retrieval and analysis, computational intelligence, and applications in bioinformatics and finance. He is a frequent consultant for biotech companies.

Dr. Zhang received Science and Technology Progress Award by State Education Commission of China, for his significant contribution in a National HighTech Project, in 1994. He is a registered Professional Engineer, ON, Canada. $\mathrm{He}$ is the publicity co-chair for ICME' 06 and program co-chair for ICIC' 05.

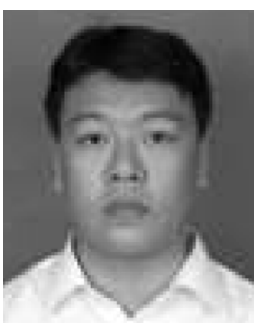

Kan $\mathbf{L i}$ received the B.Sc. degree in electrical engineering from University of Science and Technology of China, Hefei, China, in 1998 and the M. Sc. degree in electrical and computer engineering from Ryerson University, Toronto, ON, Canada, in 2004.

He is currently holding Technical Manager position at Cantata Technology, Needham, MA.

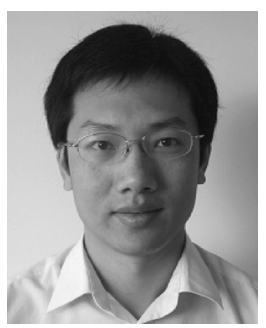

Xiaofeng Wang received the B.E. and M.E. degrees from the Faculty of Information Engineering, Beijing University of Posts and Telecommunications, Beijing, China, in 1998 and 2001, respectively. He is currently working toward the Ph.D. degree at Ryerson University, Toronto, ON, Canada.

His research interests include multimedia data hiding, information forensic, and multimedia data fusion and mining. 